\title{
DECRASE IN CEREBRAL BLOOD FLOW DURING MAXIMAL HANDGRIP ISOMETRIC CONTRACTION - A BRIEF REPORT
}

\author{
Vedran Hadžićí, Lana Ružić², Branka Matković , Bojana Žvan³, Stanko Štuhec', \\ Marjan Zaletel ${ }^{3}$, and Milan Čoh ${ }^{1}$ \\ ${ }^{1}$ University of Ljubljana, Faculty of Sport, Ljubljana, Slovenia \\ ${ }^{2}$ University of Zagreb, Faculty of Kinesiology, Zagreb, Croatia \\ ${ }^{3}$ University Medical Centre Ljubljana, Division of Neurology, Ljubljana, Slovenia
}

Preliminary communication

DOI $10.26582 / \mathrm{k} .53 .2 .8$

\begin{abstract}
:
Findings of the studies on the relationship between isometric handgrip contraction and changes in cerebral blood flow (CBF) are contradictory. The aim of the study was to evaluate CBF changes during one minute of a maximal handgrip isometric contraction. Our main hypothesis was that the maximal handgrip test will cause a decrease in CBF related to the decrease in handgrip strength. The study protocol included a transcranial Doppler ultrasound measurement of middle cerebral arterial mean flow velocity (Vmean) with the concomitant measurement of the sustained maximal handgrip strength over a period of one minute in 12 healthy male subjects. The main findings indicate that a maximal handgrip exercise causes a significant decrease in cerebral blood flow that is slightly more prominent on the contralateral side. This decrease is accompanied by a significant but transient increase in heart rate and also by an important (16\%) increase in mean arterial pressure. The maximal isometric contraction may result in an considerable decrease in cerebral blood flow that in certain cases may become even clinically relevant. In the light of our findings, we suggest that maximal isometric contractions should be better avoided as a therapeutic tool and that isometric exercises of intensities up to $60 \%$ of maximal voluntary contraction are better used.
\end{abstract}

Key words: neurophysiology, muscle function, exercise prescription

\section{Introduction}

Cerebral blood flow (CBF) is considered to remain relatively constant during exercise (Ogoh \& Ainslie, 2009), although several studies have shown that during rhythmic handgrip exercise at the intensities of up to $60 \%$ of maximal voluntary contraction (MVC) there is a contralateral increase in CBF (Jorgensen, Perko, Payne, \& Secher, 1993; Ono, Hasegawa, Hori, Nokubi, \& Hamasaki, 2007), detected by the transcranial Doppler (TCD) ultrasound as an increase in flow velocities. Changes in the middle cerebral arterial blood flow velocity are unrelated to changes in muscle sympathetic activity, implying that sympathetic nerve activity has little effect on medium-sized arteries such as the middle cerebral artery (Hartwich, Fowler, Wynn, \& Fisher, 2010; Querido \& Sheel, 2007).

However, some studies suggested that under certain conditions (e.g. hyperventilation and high intensity of exercise) (Imms, Russo, lyawe, \& Segal, 1998) there could be a decrease in CBF during the handgrip exercise. It was hypothesized that this decrease could be related to alterations in partial pressure of carbon dioxide $\left(\mathrm{PCO}_{2}\right)$ and decreased responsiveness of cerebral arteries to sympathetic stimulation (Ainslie, Ashmead, Ide, Morgan, \& Poulin, 2005). During a strenuous exercise no increase in cerebral vascular resistance is expected to assure constant CBF; instead, $\mathrm{CBF}$ can decrease leading to an inadequate oxygen delivery to the brain and possibly contributing to the development of central fatigue (Nybo \& Rasmussen, 2007; Ogoh \& Ainslie, 2009).

Based on these findings the designed study protocol included a transcranial Doppler ultrasound measurement of middle cerebral arterial mean blood flow velocity (Vmean) with the concomitant measurement of the sustained maximal handgrip strength over a period of one minute. The main hypothesis was that the maximal handgrip exercise will cause a decrease in CBF related to the decrease in handgrip strength. To our knowledge, this was the first study to investigate such a relationship. 


\section{Methods}

\section{Study design}

This was a controlled laboratory study. During the experiment, the cerebral blood flow and the dominant hand handgrip strength in 12 healthy subjects were concomitantly measured.

\section{Participants}

Twelve young men (age $21 \pm 3$ years, body mass $69.5 \pm 11.5 \mathrm{~kg}$, body height $172.8 \pm 13.8 \mathrm{~cm}$ ) volunteered to participate in the study. The Ethical Board of the Faculty of Sport in Ljubljana approved the study. Each participant signed a consent form after the purpose of the study and study protocol, as well as small potential risks, had been explained. All participants were right-handed.

\section{Measurement of middle cerebral arterial mean blood flow velocity}

All participants were subjected to a colourcoded duplex Doppler sonography of the carotid and vertebral arteries to exclude hemodynamically lesions in carotids (Zvan, Zaletel, Pretnar Oblak, Pogacnik, \& Kiauta, 2003) between 8 and 10 a.m. The temperature in the laboratory was maintained between $22^{\circ} \mathrm{C}$ and $24^{\circ} \mathrm{C}$. In preparation for the test, each subject rested for 10-15 min in a quiet room to achieve sufficient mental and physical calm. The experiment consisted of a 2-minute baseline period followed by a handgrip test with maximal strength.

The subjects were instructed to breathe normally during the handgrip and not to hyperor hypo-ventilate. The mean arterial velocity (MCA) was recorded bilaterally in the trunks of both middle cerebral arteries through the temporal acoustic windows at a depth of 50 to $54 \mathrm{~mm}$. Two $2 \mathrm{MHz}$ probes were prevented from moving by a mechanical probe holder (Doppler-Box, Sipplingen, Germany). Throughout the procedure, the mean arterial blood pressure (MAP) and heart rate (HR) were measured continuously using a non-invasive wrist tonometer (Colin CBM-7000, KomakiCity, Japan). The $\mathrm{PCO}_{2}$ was not followed by an infrared capnograph because it was reported to be too distractible for subjects in several previous pilot trials. The MCA, HR, and MAP values were exported by the TCD program (TCD Multi-Dop X4, DWL, Sipplingen, Germany) in Microsoft Excel format for further analysis. All measurements were carried out by a single experienced investigator.

\section{Handgrip strength testing}

The handgrip test was performed using the scientific handgrip dynamometer (Noraxon, Chattanooga, USA) that measures grip strength with the strain-gauge based isometer sensor (operational range 0 to $889 \mathrm{~N}$, sensitivity $25 \mathrm{mV}$ ) and has a direct transmission system (DTS). Subjects were tested in the supine position with their elbows flexed at $90^{\circ}$ and their forearms in the neutral position and only the dominant (right in all cases) hand was tested. The dominant hand was defined as the hand used for writing. The data from the dynamometer were directly exported to Microsoft Excel for further analysis. The single experienced investigator carried out all the measurements.

\section{Data analyses}

A diagram was plotted on a synchronized 60 second time scale to show the relationship between handgrip strength and median cerebral artery flow velocity (left - MCAL and right - MCAR).

The linear regression model was set with the MCAL and MCAR as the dependent variables and the dominant hand handgrip force as an independent predictor. Following that, three-time periods were defined: beginning (1-20 seconds), middle (21-40 seconds), and final (41-60 seconds) period. A one-way analysis of variance with the post-hoc Bonferroni correction was used to evaluate the differences among the periods.

All statistical analyses were performed using IBM SPSS Statistics for Windows (version 21.0; Armonk, NY, USA). The significance threshold was set at .05 .

\section{Results}

During one minute of the sustained isometric handgrip exercise, there was a significant decrease in the handgrip strength and CBF in both the left and right middle cerebral artery blood flow (Figure 1).

At the same time, there was a significant increase in mean arterial pressure (MAP) and a transient initial increase in heart rate (HR) (Figure 2).

The linear regression model was statistically significant for both the MCAL $(\mathrm{F}=150.83 ; \mathrm{p}<.001)$ and MCAR ( $\mathrm{F}=49,62 ; \mathrm{p}<.001)$. The decrease in handgrip strength explained $45 \%$ and $72 \%$ of the decrease in CBF through the right and left middle cerebral artery, respectively (Figure 1).

The changes in $\mathrm{CBF}$, heart rate, and MAP through the three consecutive time intervals are depicted in Figure 3. It is visible that CBF through the left and right middle cerebral artery initially decreased by $6 \%(\mathrm{p}<.001$ for both conditions) and in the case of the left (contralateral) cerebral artery there was a further $3 \%(\mathrm{p}=0.003)$ decrease in the last 20 seconds of the maximal handgrip exercise. This was not true for the right (ipsilateral) middle cerebral artery where, in the last 20 seconds, the CBF slightly but not significantly ( $p>05$ ) increased by $1 \%$. It is also shown that heart rate response increased significantly within the first 20 seconds ( $9 \%$ increase, $p=.001$ ), but when a new stationary state was achieved, heart rate decreased 


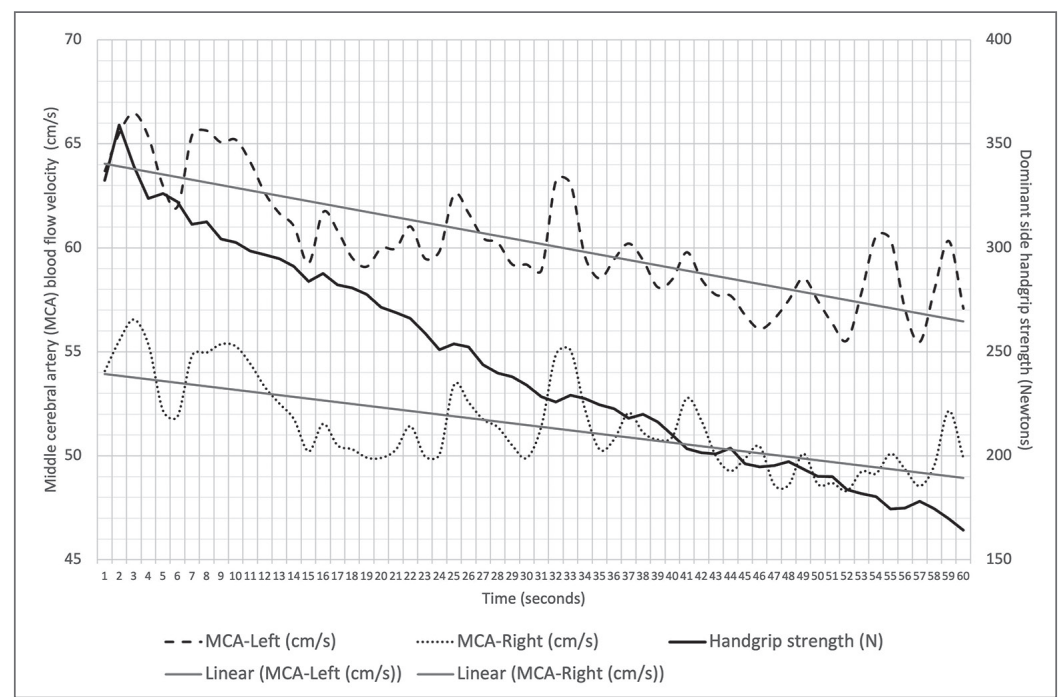

Note. Gray lines are linear trend lines for the left and right middle cerebral artery blood flow velocity over time.

Figure 1. Changes of cerebral blood flow and handgrip strength during 60 seconds of the maximal handgrip test.

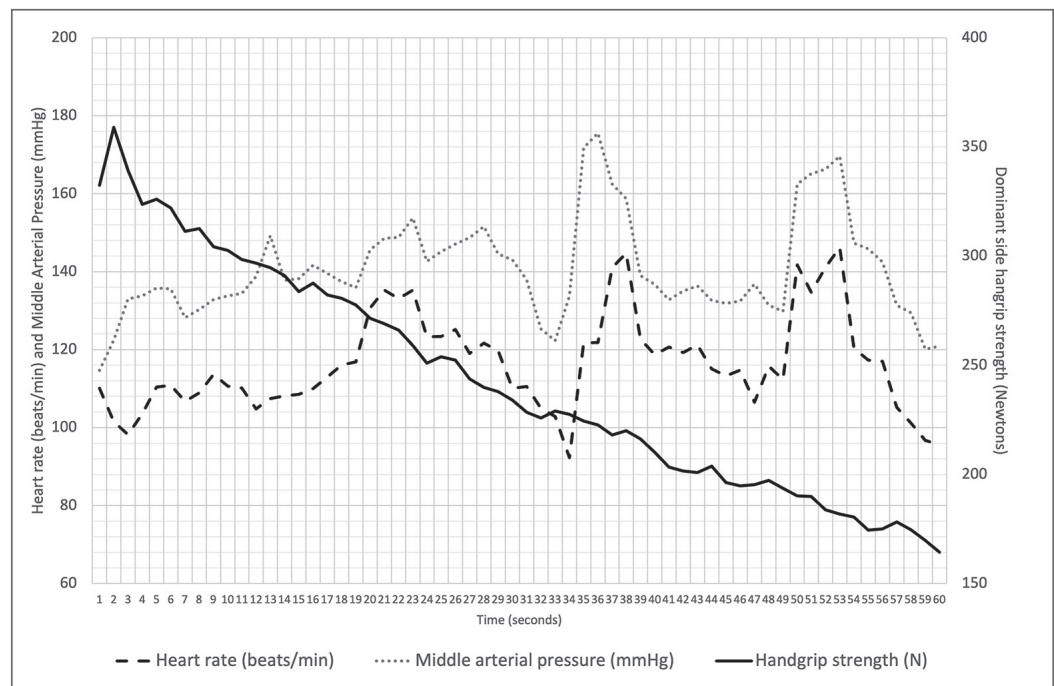

Figure 2. Changes in heart rate, mean arterial pressure, and dominant side handgrip strength during 60 seconds of the maximal handgrip test.

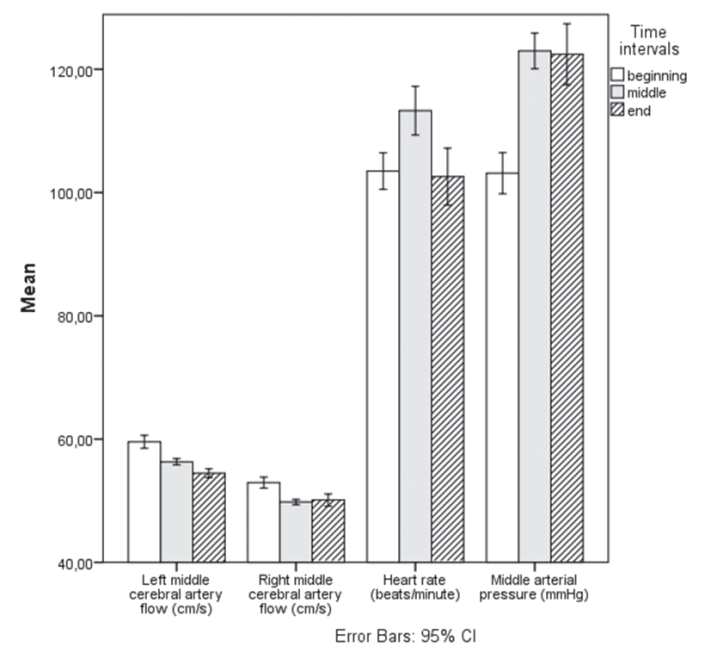

Figure 3. Changes in cerebral blood flow, heart rate, and mean arterial pressure over the three consecutive time periods of 20 seconds of the maximal handgrip test. by $10 \%(\mathrm{p}<.001)$, so that overall change of heart rate between the beginning and the end was only $1 \%$ and not significant $(\mathrm{p}>05)$. At the same time, mean arterial pressure significantly increased by $16 \%(\mathrm{p}<.001)$ and remained at that level through the whole experiment, compensating probably for the decrease in $\mathrm{CBF}$ as explained above.

\section{Discussion and conclusions}

The main findings of the study indicate that a maximal handgrip exercise might cause a significant decrease in cerebral blood flow that is slightly more prominent on the contralateral side. This decrease is accompanied by a significant but transient increase in heart rate and also by an important $(16 \%)$ increase in mean arterial pressure.

Several previous studies (Imms, et al., 1998; Ishii \& Nishida, 2013; Yamaguchi, Kashima, 
Fukuba, \& Hayashi, 2014) indicated that isometric handgrip caused an increase in $\mathrm{CBF}$, but it should be emphasized that in all those studies the handgrip was not performed with maximum effort but at a certain level of maximal voluntary force (usually around $30 \%$ ). It seems that muscle unit recruitment in those studies was probably not ample enough to require a very increased muscle blood flow, e.g. the redistribution of circulation that could result in a decreased CBF. In the present study, the handgrip was performed with maximal effort, which may be the origin of divergent results. Based on that, it could be hypothesized that the response of CBF depends on the intensity of handgrip exercise as it was suggested by Ogoh and Ainslie (2009) who concluded that exercise intensity increments of up to $60 \%$ of maximal oxygen uptake might induce elevations in CBF, after which CBF decreased toward baseline values most likely due to lower $\mathrm{PCO} 2$ via hyperventilation-induced cerebral vasoconstriction. As $\mathrm{PCO} 2$ was not measured in the present experiment, it would be speculative to discuss its possible role in the observed decrease in CBF. The other explanation might be that the maximal handgrip also induces a kind of Valsalva manoeuvre (VM). It is known that not only isometric but also isotonic contractions that are performed above $80 \%$ of $1-\mathrm{RM}$ elicit Valsalva manoeuvre, which then produces mechanical effects on the cerebral circulation in humans despite the cerebral autoregulatory responses. During VM there is a reduction in filling of atria and a decrease in stroke volume, which might lead to a transient $\mathrm{CBF}$ velocity reduction during VM. For that reason, maximal isometric contractions should be avoided in strength training of hypertensive patients, even if those are under therapy or have been well-regulated otherwise.

The contralateral effect that was observed, where a decrease in $\mathrm{CBF}$ was higher in the left middle cerebral artery $(9 \%)$ compared to the right middle cerebral artery $(6 \%)$, was expected, and in concordance with previous studies (Jorgensen, et al., 1993; Ono, et al., 2007).

The results show that maximal handgrip caused considerable increase in mean arterial pressure.
This finding was also observed in other studies (Yamaguchi, et al., 2014), regardless of the intensity of isometric handgrip exercise. The heart rate response was transient and after the initial raise, the values normalized toward the end of the experiment, unlike the MAP that remained elevated throughout the experiment.

From the clinical standpoint, we believe that our findings could be important for therapeutic exercise prescription. It seems that the safety of the isometric exercise is related to the intensity of exercise. Rhythmic handgrip exercises at the intensities of $20-50 \%$ of maximal voluntary contraction (Millar, McGowan, Cornelissen, Araujo, \& Swaine, 2014) may have beneficial effects on the reduction of blood pressure, and intensities up to $60 \%$ could improve CBF (Ogoh \& Ainslie, 2009). Nevertheless, the higher intensities and especially forceful maximal isometric contractions may have a negative influence on those parameters and should be avoided especially in diabetes mellitus type II patients because studies have shown that dynamic cerebral blood flow autoregulation is blunted with high-intensity isometric contractions. Patients who participate in high-intensity isometric contractions could therefore be more vulnerable to cerebral events during these tasks (Vianna, et al., 2015). The relationship between the intensity of isometric exercise and CBF response is not a new concept, as some older studies have suggested that the magnitude of insular activation varies with the intensity of exercise, which may be further related to the level of perceived effort or central command (Williamson, McColl, Mathews, Ginsburg, \& Mitchell, 1999).

The maximal isometric contraction might result in an important transient decrease in cerebral blood flow that in certain cases may become even clinically relevant and lead to poor perfusion of the brain. It might be even suggested that the maximal isometric contractions should be better avoided as a therapeutic tool, and in some subjects even as a diagnostic tool. As a therapeutic exercise, the isometric exercises at intensities up to $60 \%$ of maximal voluntary contraction would be advised. 


\section{References}

Ainslie, P.N., Ashmead, J.C., Ide, K., Morgan, B.J., \& Poulin, M.J. (2005). Differential responses to CO2 and sympathetic stimulation in the cerebral and femoral circulations in humans. Journal of Physiology, 566(2), 613-624. doi: 10.1113/jphysiol.2005.087320

Hartwich, D., Fowler, K.L., Wynn, L.J., \& Fisher, J.P. (2010). Differential responses to sympathetic stimulation in the cerebral and brachial circulations during rhythmic handgrip exercise in humans. Experimental Physiology, 95(11), 1089-1097. doi: 10.1113/expphysiol.2010.054387

Imms, F.J., Russo, F., Iyawe, V.I., \& Segal, M.B. (1998). Cerebral blood flow velocity during and after sustained isometric skeletal muscle contractions in man. Clinical Science (London), 94(4), 353-358.

Ishii, H., \& Nishida, Y. (2013). Effect of lactate accumulation during exercise-induced muscle fatigue on the sensorimotor cortex. Journal of Physical Therapy Science, 25(12), 1637-1642. doi: 10.1589/jpts.25.1637

Jorgensen, L.G., Perko, G., Payne, G., \& Secher, N.H. (1993). Effect of limb anesthesia on middle cerebral response to handgrip. American Journal of Physiology - Heart and Circulatory Physiology, 264(2), H553-H559.

Millar, P.J., McGowan, C.L., Cornelissen, V.A., Araujo, C.G., \& Swaine, I.L. (2014). Evidence for the role of isometric exercise training in reducing blood pressure: Potential mechanisms and future directions. Sports Medicine, 44(3), 345-356. doi: 10.1007/s40279-013-0118-x

Nybo, L., \& Rasmussen, P. (2007). Inadequate cerebral oxygen delivery and central fatigue during strenuous exercise. Exercise and Sport Sciences Reviews, 35(3), 110-118. doi: 10.1097/jes.0b013e3180a031ec

Ogoh, S., \& Ainslie, P.N. (2009). Cerebral blood flow during exercise: Mechanisms of regulation. Journal of Applied Physiology, 107(5), 1370-1380. doi: 10.1152/japplphysiol.00573.2009

Ono, T., Hasegawa, Y., Hori, K., Nokubi, T., \& Hamasaki, T. (2007). Task-induced activation and hemispheric dominance in cerebral circulation during gum chewing. Journal of Neurology, 254(10), 1427-1432. doi: 10.1007/s00415007-0570-3

Querido, J.S., \& Sheel, A.W. (2007). Regulation of cerebral blood flow during exercise. Sports Medicine, 37(9), $765-782$. doi: 10.2165/00007256-200737090-00002

Vianna, L.C., Deo, S.H., Jensen, A.K., Holwerda, S.W., Zimmerman, M.C., \& Fadel, P.J. (2015). Impaired dynamic cerebral autoregulation at rest and during isometric exercise in type 2 diabetes patients. American Journal of Physiology - Heart and Circulation Physiology, 308(7), H681-H687. doi: 10.1152/ajpheart.00343.2014

Williamson, J.W., McColl, R., Mathews, D., Ginsburg, M., \& Mitchell, J.H. (1999). Activation of the insular cortex is affected by the intensity of exercise. Journal of Applied Physiology, 87(3), 1213-1219.

Yamaguchi, Y., Kashima, H., Fukuba, Y., \& Hayashi, N. (2014). Cerebral blood flow and neurovascular coupling during static exercise. Journal of Physiological Sciences, 64(3), 195-201. doi: 10.1007/s12576-014-0311-1

Zvan, B., Zaletel, M., Pretnar Oblak, J., Pogacnik, T., \& Kiauta, T. (2003). The middle cerebral artery flow velocities during head-up tilt testing in diabetic patients with autonomic nervous system dysfunction. Cerebrovascular Diseases, 15(4), 270-275. doi: 10.1159/000069496

Submitted: June 9, 2020

Accepted: July 12, 2021

Published Online First: December 3, 2021

Correspondence to:

Vedran Hadžić, M.D., Ph.D.

University of Ljubljana, Faculty of Sport

Department of Sports \& Medicine

Gortanova 22 Street, SI-1000 Ljubljana, Slovenia

E-mail: vedran.hadzic@fsp.uni-lj.si

Telephone: + 386-1-520-77-28

Fax: + 386-1-520-77-40

\section{Acknowledgments}

This study was supported by the Slovenian Research Agency through the project P5-0147. 